Article

\title{
Comparative Semen Microbiota Composition of a Stallion in a Taylorella equigenitalis Carrier and Non-Carrier State
}

\author{
Carlota Quiñones-Pérez ${ }^{1, * \mathbb{C}}$, Amparo Martínez ${ }^{2}{ }^{\mathbb{C}}$, Francisco Crespo $^{3} \mathbb{B}$ and \\ José Luis Vega-Pla ${ }^{1}$ (iD \\ 1 Laboratorio de Investigación Aplicada, Cría Caballar de las Fuerzas Armadas, \\ Carretera de Madrid Km 395A, 14014 Córdoba, Spain; jvegpla@oc.mde.es \\ 2 Genetics Department, University of Córdoba, edificio Gregor Mendel (C-5), Campus de Rabanales, \\ 14071 Córdoba, Spain; amparomartinezuco@gmail.com \\ 3 Centro Militar de Cría Caballar de Ávila, Cría Caballar de las Fuerzas Armadas, Calle Arsenio Gutiérrez \\ Palacios, s/n, 05005 Ávila, Spain; fcrecas@oc.mde.es \\ * Correspondence: cquiper@mde.es
}

Received: 2 April 2020; Accepted: 15 May 2020; Published: 17 May 2020

check for updates

Simple Summary: Contagious equine metritis carriers have become a new cause of concern in horse stud farms. Their detection can result in significant financial loss and force owners to have their animals undergo antibiotic treatment. Current research has not been able to satisfactorily explain the appearance of carriers in agent-free farms. Studies made on microbial flora have given new insights into the diagnosis and treatment of different issues in animal systems. Next-generation sequencing (NGS) is a powerful tool that can draw an accurate picture of microbial flora. Therefore, the aim of this study was to compare the seminal bacterial composition of one stallion before and after being diagnosed with Taylorella equigenitalis using NGS. Our results show that the microbial seminal flora visibly changed between the samples analyzed. Corynebacteriaceae, an opportunistic bacterial family, was more common in the infected sample. However, Porphyromonadaceae, a natural component in several tissues, was more abundant in the negative sample. Despite the constraints of a single-case study, these findings can open the door to new therapeutic tools, as flora transplants. Similarly, seminal flora analysis may foresee microbial shifts, letting practitioners take preventive actions before a potential outbreak. Furthermore, these actions would have the extra benefit of reducing the administration of antibiotics to treat an infection.

\begin{abstract}
Contagious equine metritis is receiving renewed attention due to the continuous detection of carriers in apparent agent-free farms. Interactions of Taylorella with the seminal microflora may be the plausible cause behind these spontaneous changes of the carrier state. Accordingly, the aim of this study was to compare the differences in the seminal microbiome composition of one stallion in the contagious equine metritis carrier state and non-carrier state. Samples were cryopreserved after their extraction. Cell disruption was performed by high-speed homogenization in grinding media. Bacterial families were identified via V3 amplification of the 16S rRNA gene and Ion Torrent sequencing. Only bacterial families with relative abundance above $5 \%$ were taken into consideration. The positive sample contained a strong dominance of Corynebacteriaceae $(37.75 \%)$ and Peptoniphilaceae (28.56\%). In the negative sample, the Porphyromonadaceae $(20.51 \%)$, Bacteroidaceae $(19.25 \%)$ and Peptoniphilaceae (18.57\%) families prevailed. In conclusion, the microbiome seminal composition varies when an individual carries Taylorella from when it is free of it. The wider differences were found in the Corynebacteriaceae, Porphyromonadaceae and Bacteroidaceae families. Due to the limitations of a single-case analysis, further studies are needed for a better understanding of the stallion seminal microflora interactions.
\end{abstract}


Keywords: Taylorella equigenitalis; carrier; microbiome; stallion

\section{Introduction}

Contagious equine metritis (CEM) is a concerning condition in the horse industry, as its presence in livestock reduces fertility soundness in mares and can involve commercial restrictions. Its etiologic agent is Taylorella equigenitalis, a bacteria belonging to the Actinobacteria phylum. Stallions host the agent in the distal part of the urethra, becoming a long-timer carrier [1] if they are not submitted to a disinfection standard protocol $[2,3]$.

Symptoms vary in stallions and mares. In mares, the disease manifests with endometritis, cervicitis and vaginitis of variable severity, and it sometimes appears a mucopurulent vaginal discharge. It usually leads to temporary infertility. The recovery is uneventful, but the animal becomes a carrier. There are no symptoms in stallions, but they also become carriers. Official diagnostic methods includes culture and detection of the agent by PCR from swabs taken from predilection sites [4].

Recently, attention has been brought to CEM, as new carriers have been detected in non-symptomatic farms [5]. This has raised questions about the transmission mechanisms of the bacteria [6] or even the effectiveness of diagnostic methods [7]. It has been stated that official methods may not detect low microbial concentration carriers [8,9] or that the nature of this agent makes it difficult to isolate it in culture-based methods [4]. However, next-generation sequencing studies performed in humans have shown that certain seminal microbiome compositions could lower the chance of venereal agents of surviving [10-13].

Our study presents the case of the seminal microbiome shift in a horse that underwent a spontaneous reversion from a Taylorella equigenitalis carrier state to a non-carrier one. Therefore, the aim of this study was to compare the differences in the seminal microbiome composition of one stallion in its Taylorella equigenitalis carrier and non-carrier state.

\section{Materials and Methods}

\subsection{Sample Collection}

The sample collection was performed in the Equine Breeding Centre of the Spanish Army located in Écija under the Chief of Unit authorisation. Animals are handled in accordance with Spanish law for animal welfare (Law 32/07). Animals were not submitted to extra semen extractions for our experiment sample collection nor was their daily workflow interrupted. Samples were not collected for the purpose of the study.

Samples were collected in two different batches. The first batch was composed of semen samples from six stallions. Age ranged from 8 to 18 years old. Samples were collected using an in-line gel-filter Missouri artificial vagina, with mare in oestrus as a teaser. An inner disposable plastic liner was used with each animal to avoid cross-contamination. Animals lived under the same dietary and exercise conditions, with no clinical diseases reported. Official analysis for contagious equine metritis tests [4] were negative in all cases, so animals did not receive any type of treatment. Sperm quality tests were also performed prior to seminal extraction (total volume, gel-free volume, sperm concentration, total motility and progressive motility).

Samples were cryopreserved immediately after their extraction. All samples were then analysed using next-generation sequencing following the process described below. After a beta diversity study, we found out that one of the samples was manifestly different.

This finding led to the collection of a second batch six months afterwards. It was composed of semen samples from 14 different stallions, including the animals from the first batch. Age ranged from 6 to 18 years old in this case. The collection process, environmental conditions and analysing methods post-collection were the same as the ones for the first batch. 


\subsection{Control Sample}

For each of the batches, a pattern sample (ZymoBIOMICS Microbial Community Standard ${ }^{\circledR}$, Zymo Research, Irvine, CA, USA) was included in order to evaluate the quality of the DNA extraction and its amplification.

\subsection{DNA Extraction}

DNA extraction was performed using a ZymoBIOMICS ${ }^{\circledR}$ DNA Miniprep kit (Zymo Research, Irvine, CA, USA) commercial kit. Samples were previously submitted to a combination of mechanic and enzymatic-digestion cell disruption as described by Bag [14]. Then, DNA extraction was performed following the manufacturer's instructions.

\subsection{Next-Generation Sequencing Analysis}

Amplicons were obtained using an Ion 16S Metagenomics ${ }^{\circledR}$ kit (Thermo Fisher, Waltham, MA, USA). This kit characterizes five different sets of 16S hypervariable regions, V2, V3, V4, V67 and V8. The library was constructed with an Ion Plus Fragment Library kit and amplicons were labelled with an Xpress $^{\mathrm{TM}}$ Barcode Adapters 1-16 kit. Samples were then pooled using Ion PGM ${ }^{\circledR}$ (Thermo Fisher, Waltham, MA, USA), HiQ Sequencing kit ${ }^{\circledR}$ (Thermo Fisher, Waltham, MA, USA), Ion 316 v2 BC ${ }^{\circledR}$ chip (Thermo Fisher, Waltham, MA, USA) and sequenced using the Ion $16 \mathrm{~S}^{\mathrm{TM}}$ Metagenomics Workflow in Ion Reporter ${ }^{\mathrm{TM}}$ Software (Thermo Fisher, Waltham, MA, USA).

Data analysis was performed in the Ion Reporter server system (https://ionreporter.thermofisher. com/ir/secure/home.html). $\beta$-diversity was calculated using Bray-Curtis dissimilarity analysis. Hypervariable region V3 was chosen for taxonomic bacterial identification, as it has been suggested to detect a wider range of bacterial species [15].

\subsection{Statistical Analysis}

Similarity of samples was compared calculating the Bray-Curtis dissimilarity index according to the following formula:

$$
\mathrm{BC}_{\mathrm{a}, \mathrm{b}}=1-\frac{2 \mathrm{C}_{\mathrm{a}, \mathrm{b}}}{\mathrm{S}_{\mathrm{a}}+\mathrm{S}_{\mathrm{b}}}
$$

where $a$ and $b$ are two samples; $S_{a}$ is the total number of specimens counted on site $a ; S_{b}$ is the total number of specimens counted on site $b$; and $C_{a, b}$ is the sum of the lesser counts of each bacterial family found in both sites. Value of 0 means the two samples have the same composition, and 1 means the two samples do not share any species.

Bray-Curtis indexes were then compared using Pearson's $\chi^{2}$ test. Significant values were consider when $p<0.1$.

\section{Results}

The resulting composition of the quality control had a minor variation $( \pm 5.6 \%$ maximum $)$ comparing to the composition provided by the manufacturer.

\subsection{Microbial Community Structure Differences Between Batches}

Beta diversity analysis was used to compare microbial community structure between seminal samples of different stallions at a sampling point [16]. In the first sample batch, we observed one outlier within the group (Figure 1).

We calculated the Bray-Curtis (B-C) dissimilarity index to determine the degree of similarity among samples. Significant differences were found between the Taylorella equigenitalis carrier indexes and the rest of indexes $(p<0.1)$. Results are represented in Table 1. 


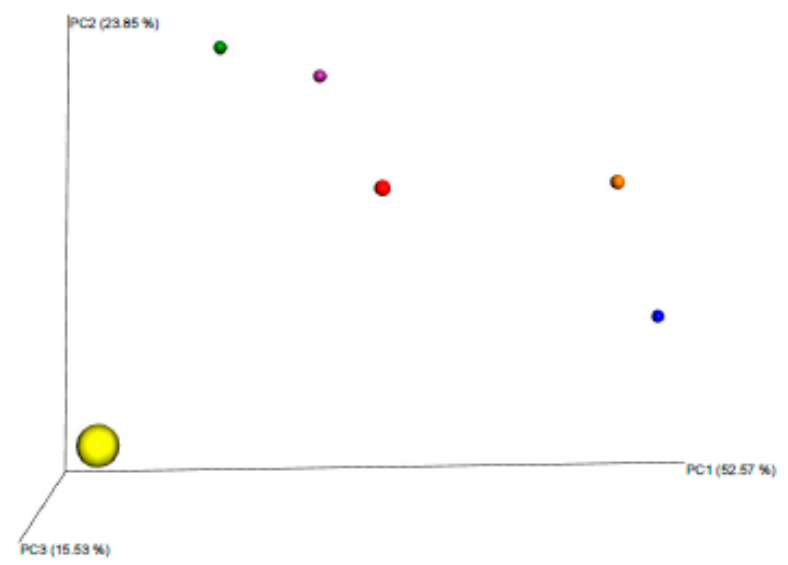

Figure 1. Family $\beta$-diversity using Bray-Curtis dissimilarity analysis of the first six-sample batch. There is a group of five samples in the upper-right area of the graph, while one sample is isolated on the down-left corner. The highlighted dot corresponds to T equigenitalis positive sample. Significant differences were found between the Taylorella equigenitalis carrier composition and the rest of samples $(p<0.1)$.

Table 1. Bray-Curtis dissimilarity indexes for the first batch.

\begin{tabular}{ccccccc}
\hline Stallions & $\mathbf{1}$ & $\mathbf{2}$ & $\mathbf{3}$ & $\mathbf{4}$ & $\mathbf{5}$ & TE+ \\
\hline 1 & - & - & - & - & - & - \\
2 & 0.40 & - & - & - & - & - \\
3 & 0.57 & 0.37 & - & - & - & - \\
4 & 0.51 & 0.55 & 0.66 & - & - & - \\
5 & 0.53 & 0.51 & 0.63 & 0.37 & - & - \\
TE $^{*}$ & 0.64 & 0.66 & 0.64 & 0.78 & 0.58 & - \\
\hline
\end{tabular}

Values near 0 means the two samples have the same composition. Values near 1 means the two samples do not share any species. Numbers represent animals. TE+: Taylorella equigenitalis carrier. ${ }^{*}$ group of samples with $p<0.1$.

This sample was reanalysed six months later following the same method within a second batch of 14 samples. This time, the former outlier sample belonged to the main group (Figure 2). Then, we also calculated the B-C dissimilarity index for this batch (Table 2). This time, no significant differences were found between samples $(p>0.1)$.

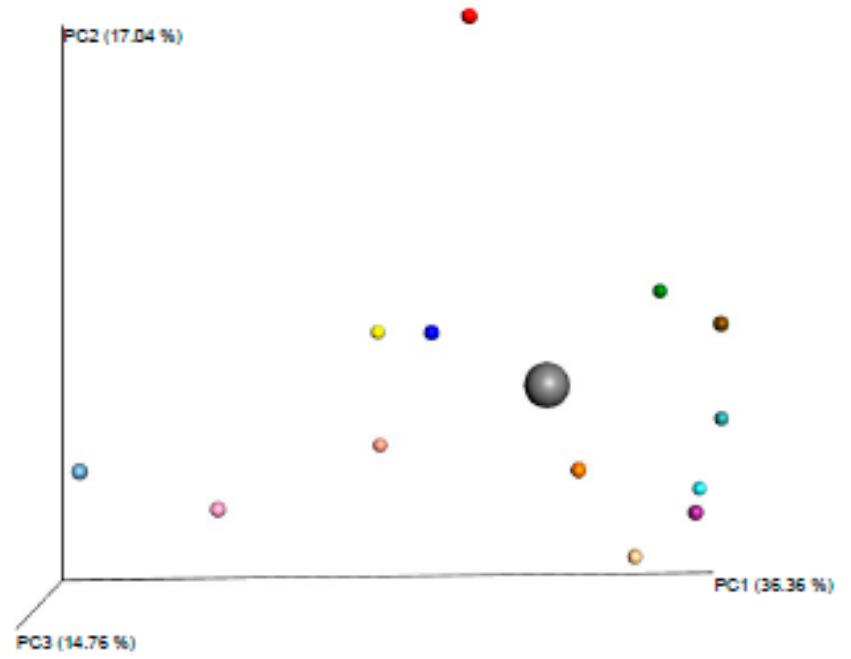

Figure 2. Family $\beta$-diversity using Bray-Curtis dissimilarity analysis of the second 14-sample batch. The highlighted dot corresponds to T equigenitalis negative sample. No significant differences were found between the Taylorella equigenitalis negative sample composition and the rest of samples $(p>0.1)$. 
Table 2. Bray-Curtis dissimilarity indexes for the second batch.

\begin{tabular}{ccccccccccccccc}
\hline Stallions & $\mathbf{1}$ & $\mathbf{2}$ & $\mathbf{3}$ & $\mathbf{4}$ & $\mathbf{5}$ & $\mathbf{6}$ & $\mathbf{7}$ & $\mathbf{8}$ & $\mathbf{9}$ & $\mathbf{1 0}$ & $\mathbf{1 1}$ & $\mathbf{1 2}$ & $\mathbf{1 3}$ & TE- \\
\hline 1 & - & - & - & - & - & - & - & - & - & - & - & - & - & - \\
2 & 0.39 & - & - & - & - & - & - & - & - & - & - & - & - & - \\
3 & 0.57 & 0.37 & - & - & - & - & - & - & - & - & - & - & - & - \\
4 & 0.51 & 0.54 & 0.65 & - & - & - & - & - & - & - & - & - & - & - \\
5 & 0.54 & 0.51 & 0.61 & 0.38 & - & - & - & - & - & - & - & - & - & - \\
6 & 0.49 & 0.54 & 0.62 & 0.44 & 0.25 & - & - & - & - & - & - & - & - & - \\
7 & 0.53 & 0.42 & 0.42 & 0.67 & 0.54 & 0.51 & - & - & - & - & - & - & - & - \\
8 & 0.54 & 0.48 & 0.59 & 0.38 & 0.22 & 0.12 & 0.53 & - & - & - & - & - & - & - \\
9 & 0.45 & 0.47 & 0.51 & 0.33 & 0.15 & 0.29 & 0.44 & 0.34 & - & - & - & - & - & - \\
10 & 0.60 & 0.60 & 0.70 & 0.14 & 0.30 & 0.39 & 0.68 & 0.32 & 0.47 & - & - & - & - & - \\
11 & 0.52 & 0.53 & 0.58 & 0.49 & 0.36 & 0.30 & 0.52 & 0.28 & 0.34 & 0.42 & - & - & - & - \\
12 & 0.53 & 0.39 & 0.48 & 0.60 & 0.47 & 0.55 & 0.54 & 0.55 & 0.49 & 0.60 & 0.54 & - & - & - \\
13 & 0.45 & 0.34 & 0.50 & 0.61 & 0.47 & 0.50 & 0.34 & 0.53 & 0.37 & 0.61 & 0.51 & 0.47 & - & - \\
TE- & 0.46 & 0.46 & 0.53 & 0.65 & 0.37 & 0.45 & 0.48 & 0.45 & 0.30 & 0.56 & 0.34 & 0.47 & 0.38 & - \\
\hline
\end{tabular}

Values near 0 means the two samples have the same composition. Values near 1 means the two samples do not share any species. Numbers represent animals. TE-: stallion cleared of Taylorella equigenitalis.

\subsection{Taxonomic Composition of Seminal Samples}

A general taxonomic composition of batches 1 and 2 is represented in Figure 3. The most abundant phyla were Firmicutes and Bacteroidetes in all cases, except for the Taylorella equigenitalis carrier (TE+). The two most dominant phyla for TE+ were Firmicutes and Actinobacteria.

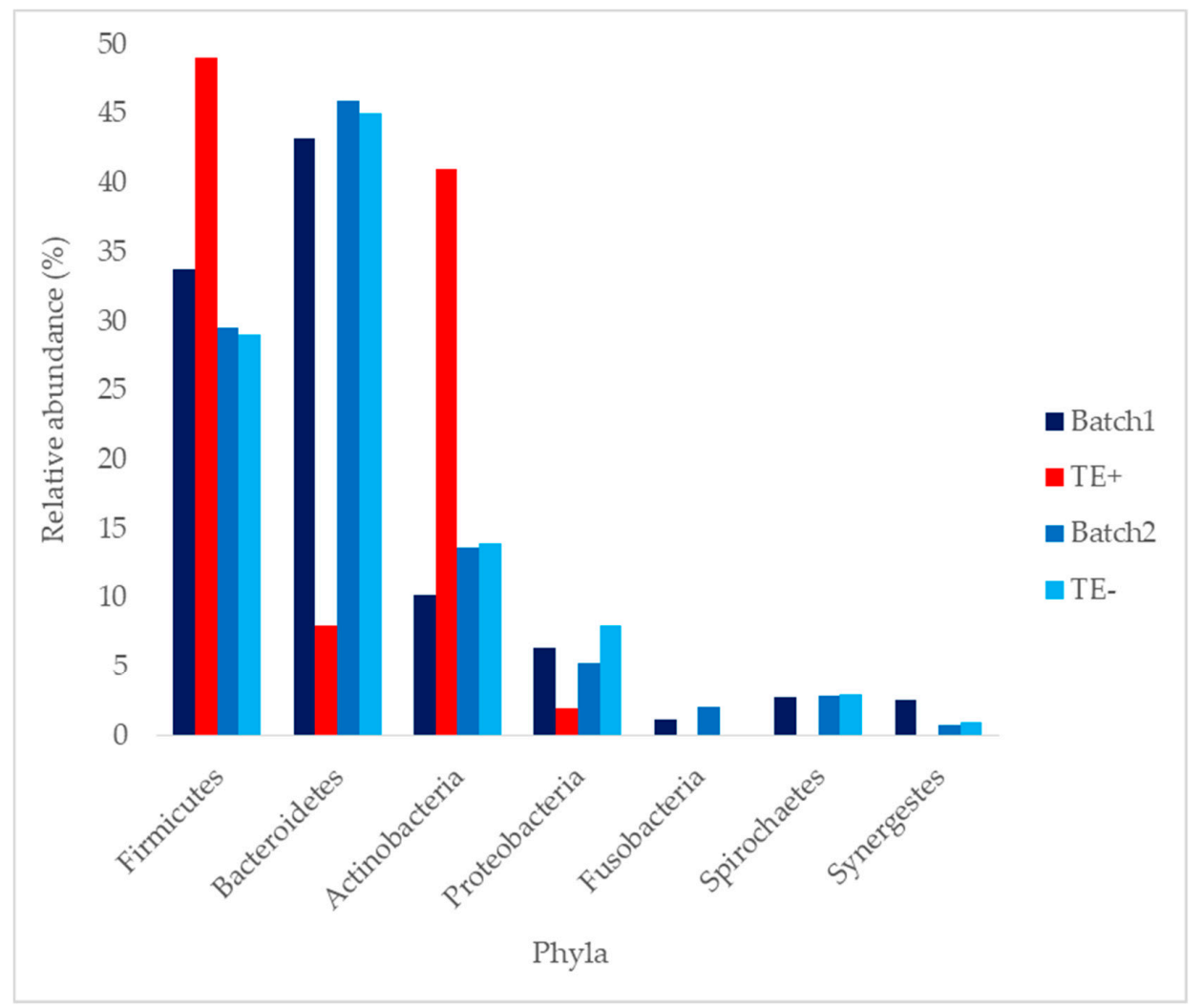

Figure 3. Mean phylum taxonomic composition of batch 1, TE+, batch 2 and TE-. It is observed how TE+ phylum composition highly varies compared with batch 1, batch 2 and TE-. TE+: Taylorella equigenitalis carrier. TE-: stallion cleared of Taylorella equigenitalis. Results are expressed as percentage (\%).

Mean values of batch 1 were 36.33\% for Firmicutes phylum, 37.33\% for Bactoroidetes; $15.33 \%$ for Actinobacteria; $5.50 \%$ for Proteobacteria; $1.50 \%$ for Fusobacteria; $2.00 \%$ for Spirochaetes; and $2.23 \%$ for 
Synergestes. Mean values of batch 2 were $29.50 \%$ for Firmicutes phylum, $45.86 \%$ for Bactoroidetes; 13.71\% for Actinobacteria; 5.27\% for Proteobacteria; $1.86 \%$ for Fusobacteria; $2.91 \%$ for Spirochaetes; and $0.89 \%$ for Synergestes.

\subsection{Comparative Taxonomic Composition of TE+ and TE-}

Figure 4 shows relative abundance at the family level in samples TE+ and TE- (stallion cleared of Taylorella equigenitalis). Only common families with a relative abundance above $5 \%$ are represented.

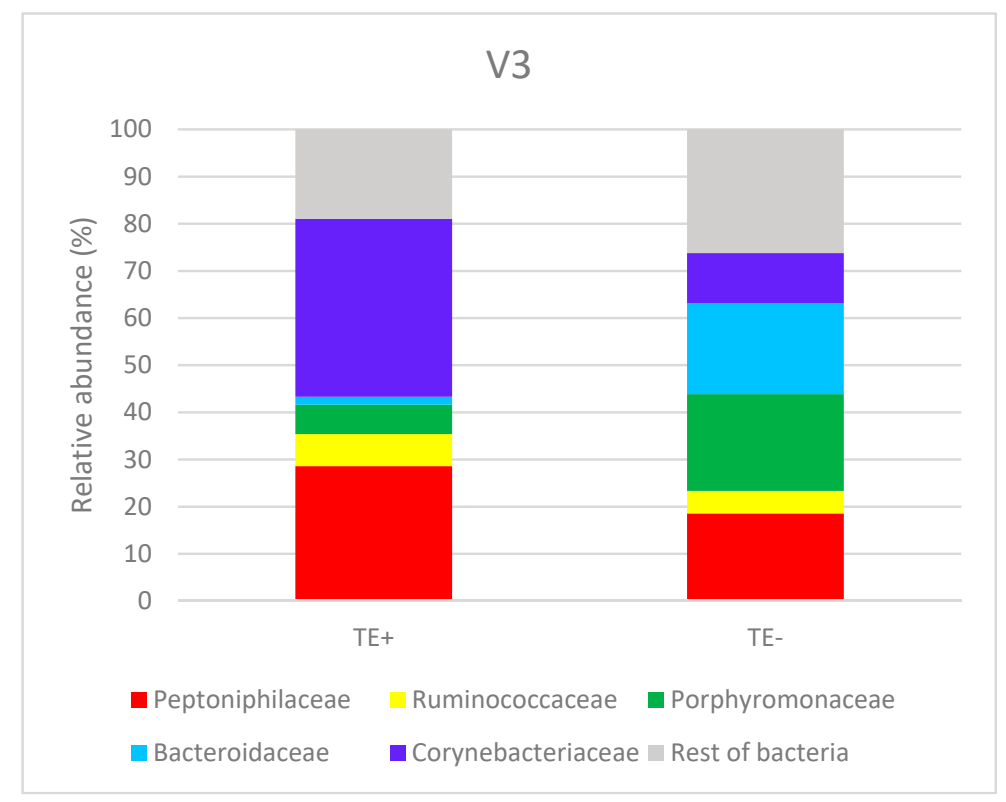

Figure 4. Comparative taxonomic composition of TE+ and TE-. The results are expressed as percentages (\%). TE + corresponds to T equigenitalis positive sample and TE- to the stallion cleared of T equigenitalis. Only common families with a relative abundance above $5 \%$ are included separately.

Taxonomic composition classification revealed that TE+ contained a $0.02 \%$ of the Alcaligenaceae family (represented by Taylorella equigenitalis species), while TE- showed no remainders of the Taylorella equigenitalis species nor its complete family.

As far as general bacterial composition is concerned, TE+ presents a microbial diversity of 32 families, with a strong dominance of Corynebacteriaceae (up to 37.75\%) and Peptoniphilaceae (28.56\%). The percentage of the following most common bacteria, Ruminococcaceae, only reaches $6.82 \%$. Regarding TE-, it contains 31 different bacteria families, with a dominance of Porphyromonadaceae (20.51\%), Bacteroidaceae (19.25\%) and Peptoniphilaceae (18.57\%). Corynebacteriaceae is the fourth most abundant family with a $10.64 \%$ presence. The family composition of TE+ also contrasts with the composition of the rest of samples. Thus, mean values for the families are represented in Table 3.

Table 3. Mean values for Porphyromonadaceae, Bacteroidaceae, Prevotellaceae and Corynebacteriaceae families of the TE+ sample and the first batch of samples without TE+.

\begin{tabular}{ccc}
\hline Bacterial families & TE + & Rest of Samples \\
\hline Porphyromonadaceae & 6.24 & $30.07 \pm 16.18$ \\
Bacteroidaceae & 1.68 & $0.80 \pm 0.60$ \\
Prevotellaceae & 0.08 & $10.72 \pm 6.43$ \\
Corynebacteriaceae & 37.75 & $7.87 \pm 2.38$ \\
\hline
\end{tabular}

Results are expressed as percentage (\%) and mean \pm standard error of the mean. TE + corresponds to Taylorella equigenitalis positive sample. 


\section{Discussion}

Our results show there is a different microbiome composition when an animal carries Taylorella equigenitalis than when it is free of the agent. It has already been proven that some microflora changes in the equine digestive tract can favor the growth of some pathogens [17-19]. In the genital tract, it has also been hypothesized that infections or strange agents may produce changes in the environment around them and, therefore, favor some bacteria families to grow while hindering others [20].

In our case, Actinobacteria phylum highly differs between TE+ and TE- (41\% vs. 14\%, respectively). The main family of this phylum, Corynebacteriaceae, has been regularly described as a normal component of seminal flora in humans [20-25] as well as in stallions [26-32]. Meanwhile, other authors maintain that it holds an opportunistic nature, and even some have related its presence with a higher caspases activity [33].

Concerning Bacteroidetes phylum, the three most common families have largely varied from the carrier situation to the non-carrier one. Mändar have already pointed out that the presence of Prevotellaceae family alone or combined with Porphyromonadaceae are associated with a higher rate of reproductive inflammatory conditions [23]. Our results may contradict this finding, as they are the predominant group in the non-carrier state, especially Porphyromonadaceae. It is relevant to say that it is not easy to compare the effect of the Bacteroidetes phylum with previous studies, as it is a difficult to culture group and most studies utilized cultured-based methods.

In Mändar [23], it was possible to divide a population in three groups according to their seminal microbiome composition, that time relating the results with their seminal quality. In our case, the division would create two groups: the Corynebacteriaceae family predominant group, representing the contagious equine metritis carriers; and the Bacteroidetes phylum predominant group for the non-carrier stallions. It would be of the utmost importance to dig deeper into the mechanisms underlying these differences, as understanding the seminal microbiome composition can open the door to a future diagnostic or even prophylactic tools.

Finally, it is necessary to indicate some strengths and limitations of this study. Next-generation sequencing overcomes limitations of culture-based diagnostic methods. It can detect fastidious-to-cultivate genera and it is less affected by contamination [34]. In spite of that, NGS also has limitations. For example, differential amplification of primers [35], DNA extraction or interpretation of data [12]. Another limitation of our study would be sample size.

\section{Conclusions}

The microbiome seminal composition varies when an individual carries Taylorella equigenitalis from when it is free of the agent. The wider differences were found in the Corynebacteriaceae family, increased in the carrier case; and the Porphyromonadaceae and Bacteroidaceae families, increased in the non-carrier case. Besides, we have observed it is possible to detect CEM carriers using NGS. However, due to the limitations of a one-subject case report, further studies are needed in order to completely comprehend the interactions that occur in the stallion seminal microflora.

Author Contributions: Conceptualization, C.Q.-P.; formal analysis, C.Q.-P.; writing-original draft preparation, C.Q.-P.; writing-review and editing, A.M., F.C. and J.L.V.-P.; supervision, A.M., F.C. and J.L.V.-P.; visualization, C.Q.-P. All authors have read and agreed to the published version of the manuscript.

Funding: This research received no external funding.

Conflicts of Interest: The authors declare no conflict of interest.

\section{References}

1. Schluter, H.; Kuller, H.J.; Friedrich, U.; Selbitz, H.J.; Marwitz, T.; Beyer, C.; Ullrich, E. Epizootiology and treatment of contagious equine metritis (CEM), with particular reference to the treatment of infected stallions. Prakt. Tierarzt 1991, 72, 503-511.

2. Timoney, P.J. Contagious equine metritis. Comp. Immunol. Microbiol. Infect. Dis. 1996, 19, 199-204. [CrossRef] 
3. Crowhurst, R.; Simpson, D.; Greenword, R.; Ellis, D. Contagious equine metritis. Vet. Rec. 1979, $104,465$. [CrossRef] [PubMed]

4. World Organisation for Animal Health. Manual of Diagnostic Tests and Vaccines of Terrestrial Animals, 8th ed.; OIE Biological Standards Commission: Paris, France, 2018; ISBN 978-92-9044-884-6.

5. Timoney, P.J. Horse species symposium: Contagious equine metritis: An insidious threat to the horse breeding industry in the United States. J. Anim. Sci. 2011, 89, 1552-1560. [CrossRef]

6. Schulman, M.L.; May, C.E.; Keys, B.; Guthrie, A.J. Contagious equine metritis: Artificial reproduction changes the epidemiologic paradigm. Vet. Microbiol. 2013, 167, 2-8. [CrossRef]

7. Matsuda, M.; Moore, J.E. Recent advances in molecular epidemiology and detection of Taylorella equigenitalis associated with contagious equine metritis (CEM). Vet. Microbiol. 2003, 97, 111-122. [CrossRef]

8. Aznai, T.; Wada, R.; Okuda, T.; Aoki, T. Evaluation of the field application of PCR in the eradication of contagious equine metritis from Japan. J. Vet. Med. Sci. 2002, 11, 999-1002.

9. Nadin-Davis, S.; Knowles, M.K.; Burke, T.; Böse, R.; Devenish, J. Comparison of culture versus quantitative real-time polymerase chain reaction for the detection of Taylorella equigenitalis in field samples from naturally infected horses in Canada and Germany. Can. J. Vet. Res. 2015, 79, 161-169.

10. Altmäe, S.; Franasiak, J.M.; Mändar, R. The seminal microbiome in health and disease. Nat. Rev. Urol. 2019, 16, 703-721. [CrossRef]

11. Witkin, S.S.; Linhares, I.M. HIV inhibition by lactobacilli: Easier in a test tube than in real life. mBio 2015, 6, e01485-01415. [CrossRef]

12. Mändar, R.; Punab, M.; Korrovits, P.; Türk, S.; Ausmees, K.; Lapp, E.; Preem, J.-K.; Oopkaup, K.; Salumets, A.; Truu, J. Seminal microbiome in men with and without prostatitis. Int. J. Urol. Off. J. Jpn. Urol. Assoc. 2017, 24, 211-216. [CrossRef] [PubMed]

13. Korhonen, C.J.; Srinivasan, S.; Huang, D.; Ko, D.L.; Sanders, E.J.; Peshu, N.M.; Krieger, J.N.; Muller, C.H.; Coombs, R.W.; Fredricks, D.N.; et al. Semen Bacterial Concentrations and HIV-1 RNA Shedding Among HIV-1-Seropositive Kenyan Men. J. Acquir. Immune Defic. Syndr. 2017, 74, 250-257. [CrossRef] [PubMed]

14. Bag, S.; Saha, B.; Mehta, O.; Anbumani, D.; Kumar, N.; Dayal, M.; Pant, A.; Kumar, P.; Saxena, S.; Allin, K.H.; et al. An Improved Method for High Quality Metagenomics DNA Extraction from Human and Environmental Samples. Sci. Rep. 2016, 6, srep26775. [CrossRef] [PubMed]

15. Chakravorty, S.; Helb, D.; Burday, M.; Connell, N.; Alland, D. A detailed analysis of 16S ribosomal RNA gene segments for the diagnosis of pathogenic bacteria. J. Microbiol. Methods 2007, 69, 330-339. [CrossRef]

16. Goodrich, J.K.; Di Rienzi, S.C.; Poole, A.C.; Koren, O.; Walters, W.A.; Caporaso, J.G.; Knight, R.; Ley, R.E. Conducting a microbiome study. Cell 2014, 158, 250-262. [CrossRef]

17. Clark, A.; Sallé, G.; Ballan, V.; Reigner, F.; Meynadier, A.; Cortet, J.; Koch, C.; Riou, M.; Blanchard, A.; Mach, N. Strongyle Infection and Gut Microbiota: Profiling of Resistant and Susceptible Horses Over a Grazing Season. Front. Physiol. 2018, 9, 272. [CrossRef]

18. Weese, J.S.; Holcombe, S.J.; Embertson, R.M.; Kurtz, K.A.; Roessner, H.A.; Jalali, M.; Wismer, S.E. Changes in the faecal microbiota of mares precede the development of post partum colic. Equine Vet. J. 2015, 47, 641-649. [CrossRef]

19. Morrison, P.K.; Newbold, C.J.; Jones, E.; Worgan, H.J.; Grove-White, D.H.; Dugdale, A.H.; Barfoot, C.; Harris, P.A.; Argo, C.M. The Equine Gastrointestinal Microbiome: Impacts of Age and Obesity. Front. Microbiol. 2018, 9, 3017. [CrossRef]

20. Liu, C.M.; Osborne, B.J.W.; Hungate, B.A.; Shahabi, K.; Huibner, S.; Lester, R.; Dwan, M.G.; Kovacs, C.; Contente-Cuomo, T.L.; Benko, E.; et al. The Semen Microbiome and Its Relationship with Local Immunology and Viral Load in HIV Infection. PLoS Pathog. 2014, 10. [CrossRef]

21. Hou, D.; Zhou, X.; Zhong, X.; Settles, M.L.; Herring, J.; Wang, L.; Abdo, Z.; Forney, L.J.; Xu, C. Microbiota of the seminal fluid from healthy and infertile men. Fertil. Steril. 2013, 100, 1261-1269. [CrossRef]

22. Ivanov, I.B.; Kuzmin, M.D.; Gritsenko, V.A. Microflora of the seminal fluid of healthy men and men suffering from chronic prostatitis syndrome. Int. J. Androl. 2009, 32, 462-467. [CrossRef] [PubMed]

23. Mändar, R.; Punab, M.; Borovkova, N.; Lapp, E.; Kiiker, R.; Korrovits, P.; Metspalu, A.; Krjutškov, K.; Nõlvak, H.; Preem, J.-K.; et al. Complementary seminovaginal microbiome in couples. Res. Microbiol. 2015, 166, 440-447. [CrossRef] [PubMed]

24. Jarvi, K.; Lacroix, J.M.; Jain, A.; Dumitru, I.; Heritz, D.; Mittelman, M.W. Polymerase chain reaction-based detection of bacteria in semen. Fertil. Steril. 1996, 66, 463-467. [CrossRef] 
25. Al-Kass, Z.; Spergser, J.; Aurich, C.; Kuhl, J.; Schmidt, K.; Morrell, J.M. Effect of presence or absence of antibiotics and use of modified single layer centrifugation on bacteria in pony stallion semen. Reprod. Domest. Anim. 2019, 54, 342-349. [CrossRef]

26. Varela, E.; Rey, J.; Plaza, E.; Muñoz de Propios, P.; Ortiz-Rodríguez, J.M.; Álvarez, M.; Anel-López, L.; Anel, L.; De Paz, P.; Gil, M.C.; et al. How does the microbial load affect the quality of equine cool-stored semen? Theriogenology 2018, 114, 212-220. [CrossRef]

27. Althouse, C.; Skaife, J.; Loomis, P. Prevalence and types of contaminant bacteria in extended, chilled equine semen. Anim. Reprod. Sci. 2010, 121, 224-225. [CrossRef]

28. Madsen, M.; Christensen, P. Bacterial flora of semen collected from Danish warmblood stallions by artificial vagina. Acta Vet. Scand. 1995, 36, 1-7.

29. Pickett, B.W.; Voss, J.L.; Jones, R.L. Control of bacteria in stallions and their semen. J. Equine Vet. Sci. 1999, 19, 424-469. [CrossRef]

30. Varner, D.D.; Scanlan, C.M.; Thompson, J.A.; Brumbaugh, G.W.; Blanchard, T.L.; Carlton, C.M.; Johnson, L. Bacteriology of preserved stallion semen and antibiotics in semen extenders. Theriogenology 1998, 50, 559-573. [CrossRef]

31. Hannachi, H.; Elloumi, H.; Hamdoun, M.; Kacem, K.; Zhioua, A.; Bahri, O. Bacteriospermia: Effects on semen parameters. Gynecol. Obstet. Fertil. Senol. 2018, 46, 518-523. [CrossRef]

32. Meštrović, T.; Bedenić, B.; Wilson, J.; Ljubin-Sternak, S.; Sviben, M.; Neuberg, M.; Ribić, R.; Kozina, G.; Profozić, Z. The impact of Corynebacterium glucuronolyticum on semen parameters: A prospective pre-post-treatment study. Andrology 2018, 6, 223-229. [CrossRef] [PubMed]

33. Ortega-Ferrusola, C.; González-Fernández, L.; Muriel, A.; Macías-García, B.; Rodríguez-Martínez, H.; Tapia, J.A.; Alonso, J.M.; Peña, F.J. Does the microbial flora in the ejaculate affect the freezeability of stallion sperm? Reprod. Domest. Anim. Zuchthyg. 2009, 44, 518-522. [CrossRef] [PubMed]

34. Namdari, S.; Nicholson, T.; Abboud, J.; Lazarus, M.; Ramsey, M.L.; Williams, G.; Parvizi, J. Comparative study of cultures and next-generation sequencing in the diagnosis of shoulder prosthetic joint infections. J. Shoulder Elbow Surg. 2019, 28, 1-8. [CrossRef] [PubMed]

35. Lambert, J.A.; Kalra, A.; Dodge, C.T.; John, S.; Sobel, J.D.; Akins, R.A. Novel PCR-Based Methods Enhance Characterization of Vaginal Microbiota in a Bacterial Vaginosis Patient before and after Treatment. Appl. Environ. Microbiol. 2013, 79, 4181-4185. [CrossRef] 